\title{
UNSTABLE HOMEOMORPHISMS
}

\author{
W. R. UTZ
}

1. Definitions. Let $f$ be a homeomorphism of a metric space $X$ (with metric $d$ ) onto itself. $f$ will be called unstable on $X$ provided there is a number $\delta(f, X)>0$ (called an instability constant) such that corresponding to each pair of distinct points $x, y$ of $X$, there is an integer $n(x, y)$ for which

$$
d\left(f^{n}(x), f^{n}(y)\right)>\delta .
$$

As an example of an unstable homeomorphism let $X$ be the Euclidean plane. Select a point $O$ of $X$. Define $f(O)=O$ and if $O \neq p \in X$, define $f(p)=q$ where $q$ is the point of the ray $O p$, with end point $O$, such that $d(O, q)=2 d(O, p)$. Now $f$ is unstable, with $\delta$ arbitrary, since if $d(x, y)=r$,

$$
d\left(f^{n}(x), f^{n}(y)\right)=2^{n} \cdot r .
$$

Let $M$ be a metric space and let $\phi(M)=M$ be a homeomorphism. $O(m) \equiv \sum_{-\infty}^{+\infty} \phi^{i}(m)$ is called the orbit of $m \in M$ under $\phi, O_{-}(m)$ $\equiv \sum_{-\infty}^{-1} \phi^{i}(m)$ is called the negative semi-orbit of $m \in M$ under $\phi$, and $O_{+}(m) \equiv \sum_{0}^{+\infty} \phi^{i}(m)$ is called the positive semi-orbit of $m \in M$ under $\phi$. The point $p$ is said to be an $\alpha$-limit point ( $\omega$-limit point) of the orbit $O$ if there exists a point $x \in O$ and a sequence of integers $n_{1}>n_{2}>\ldots$ $\left(n_{1}<n_{2}<\cdots\right)$ such that $\lim \phi^{n_{i}}(x)=p$. The set of all $\alpha$-limit points and the set of all $\omega$-limit point of an orbit $O$ are çlosed sets which are invariant under $\phi$ (cf. $[3, \S 2]) .^{1}$

$O(m)$ will be called positively (negatively) asymptotic to a set $A \subset M$ under $\phi$ if, given $\epsilon>0$, there exists an integer $N(\epsilon)$ such that $n>N$ $(n<N)$ implies $\phi^{n}(m) \in V_{\epsilon}(A)$, the $\epsilon$-neighborhood of $A$. Let $x$ and $y$ be distinct points of $M . O(x)$ and $O(y)$ will be called positively (negatively) asymptotic if, given $\epsilon>0$, there exists an integer $N$ such that $n>N(n<N)$ implies

$$
d\left(\phi^{n}(x), \phi^{n}(y)\right)<\epsilon .
$$

2. Instability and asymptoticity. A space is said to be dense-initself if every point of the space is a limit point of points of the space. Unless otherwise qualified $X$, in this section, shall denote a compact metric space that is dense-in-itself and $f(X)=X$ shall be an unstable

Presented to the Society, September 2, 1949; received by the editors October 1, 1949.

1 Numbers in brackets refer to the references cited at the end of the paper. 
homeomorphism with instability constant $\delta$.

(2.1) THEOREM. $X$ contains a pair of points whose orbits are asymptotic in at least one sense.

Proof. Consider the product space $X^{2} \equiv X \times X$ and the homeomorphism $g\left(X^{2}\right)=X^{2}$ defined as $g\left(x_{1} \times x_{2}\right)=f\left(x_{1}\right) \times f\left(x_{2}\right), x_{1}, x_{2} \in X$. If $z_{1}=x_{1} \times y_{1}$ and $z_{2}=x_{2} \times y_{2}$ are elements of $X^{2}$, let

$$
\rho\left(z_{1}, z_{2}\right)=d\left(x_{1}, x_{2}\right)+d\left(y_{1}, y_{2}\right) \text {. }
$$

With this definition, $X^{2}$ becomes a metric space. ${ }^{2}$

If $D$ denotes all elements of $X^{2}$ of the form $x \times x, x \in X$, then $D$ is compact and invariant under $g$. Let $U$ be the $\delta$-neighborhood of $D$ in $X^{2}$. If $z=x \times y$ is a point of $U$, then $\rho(z, D)<\delta$, that is, $d(x, p)$ $+d(y, p)<\delta$ for some $p \in X$. However, $d(x, y) \leqq d(x, p)+d(y, p)<\delta$, hence $d(x, y)<\delta$. Thus, since $f$ is unstable on $X$, the only points of $X^{2}$ whose orbits (under $g$ ) lie in $U$ are points of $D$.

Let $N(D)$ be a neighborhood of $D$ in $X^{2}$ such that its closure, $\bar{N}(D)$, is a subset of $U$ and let $\left\{z_{i}\right\}$ be a sequence of points of $X^{2}-D$ such that $z_{i} \rightarrow z \in D$. Since $\bar{N}(D) \subset U$ and $f$ is unstable on $X$, no orbit $O\left(z_{i}\right), i=1,2, \cdots$, can be entirely in $\bar{N}(D)$. Thus for each $i$ there is an integer $m_{i}$ such that

$$
\mathrm{g}^{m_{i}}\left(z_{i}\right) \notin \bar{N}(D) .
$$

For each $i$ we can assume that $m_{i}$ has the smallest absolute value for any integer with the stated property. There are either an infinite number of the collection $\left\{m_{i}\right\}$ that are positive or an infinite number that are negative, or both. Suppose that there are an infinite number of positive elements of the sequence and let $\left\{m_{i}\right\}$ denote this class. In case there are an infinite number of negative elements of the original sequence, the proof is similar. The collection can be considered as ordered so that the elements increase with their subscripts. There exists a point $s \in X^{2}-N(D)$ such that for some subcollection of $\left\{m_{i}\right\}$

$$
g^{m_{i}}\left(z_{i}\right) \rightarrow s .
$$

Hereafter, $\left\{m_{i}\right\}$ is to denote this subsequence of the initial sequence.

The negative semi-orbit of $s$ lies in $\bar{N}(D)$ since

$$
g^{m_{i}-m}\left(z_{i}\right)=g^{-m}\left(g^{m_{i}}\left(z_{i}\right)\right) \rightarrow g^{-m}(s)
$$

and for $i$ sufficiently large and $m>0$,

${ }^{2} g\left(X^{2}\right)=X^{2}$ inherits instability from $f$ with the same instability constant. 


$$
g^{m_{i}-m}\left(z_{i}\right) \in \bar{N}(D) .
$$

Let $O$ denote the orbit of $s$. Since $\bar{N}(D)$ is closed, $O_{\alpha}$, the set of $\alpha$-limit points of $O$, is in $\bar{N}(D)$. Moreover $O_{\alpha}$ is invariant and if $O$ is not negatively asymptotic to the set $D$, there exists a point $\alpha_{0}$ in $O_{\alpha}$ but not in $D$ such that

$$
O\left(\alpha_{0}\right) \subset O_{\alpha} \subset \bar{N}(D) \subset U .
$$

As was remarked, an orbit remains in $U$ only if it is the orbit of a point of $D$; thus we conclude that $O$ is negatively asymptotic to $D$.

If $s=x \times y$, then for $\epsilon>0$ there exists an $N$ such that for $n<N$, $\rho\left(g^{n}(s), D\right)<\epsilon$. This implies $d\left(f^{n}(x), f^{n}(y)\right)<\epsilon$ and the proof of the theorem is complete.

An unstable homeomorphism followed by an unstable homeomorphism is not necessarily unstable. However, under some conditions a combination of homeomorphisms is always unstable. In order to continue the investigation of asymptotic orbits we digress to prove the following theorem.

(2.2) TheOREM. Let $X$ be compact and metric and let $f(X)=X$ be an unstable homeomorphism. For any integer $m \neq 0, f^{m}(X)=X$ is unstable.

Proof. We first recall that if $X$ is compact and metric and $M(X)$ $=X$ is a homeomorphism, then given $\xi>0$ there exists $\eta>0$ such that $d(x, y)>\xi$ implies $d(M(x), M(y))>\eta$. Now, let $\phi(X) \equiv f^{m}(X)$ and consider the homeomorphisms $f^{i}(X), i= \pm 1, \pm 2, \cdots, \pm m$. By the remark above, there exist numbers $\eta_{i}>0(i= \pm 1, \pm 2, \cdots, \pm m)$ such that $d(x, y)>\delta$ implies $d\left(f^{i}(x), f^{i}(y)\right)>\eta_{i}$, for all $x, y \in X$. We shall show that $\min \left\{\eta_{i}\right\}$ is an instability constant for $\phi(X)$. If $x$ and $y$ are distinct points of $X$, there exists an $n$ such that

$$
d\left(f^{n}(x), f^{n}(y)\right)>\delta .
$$

There exists an integer $r$ such that $0<|r m-n| \leqq m$ and if $i$ is taken as $r m-n$, then

$$
f^{i}\left(f^{n}(s)\right)=f^{r m}(s)=\phi^{r}(s), \quad s \in X \cdot
$$

Since $d\left(f^{n}(x), f^{n}(y)\right)>\delta$, by the remark above $d\left(\phi^{r}(x), \phi^{r}(y)\right)$ $>\min \left\{\eta_{i}\right\}$ and hence $\phi(X)$ is unstable with instability constant $\min \left\{\eta_{i}\right\}$.

(2.3) Lemma. If $x \in X$ is a fixed point of $f(X)=X$, then there exists a point of $X$ whose orbit is asymptotic to $x$ in at least one sense.

Proof. Let $U$ be the $\delta$-neighborhood of $x$ and let $\left\{x_{i}\right\}$ be a sequencc 
of points of $X-(x)$ for which $\lim x_{i}=x$. Let $N \subset U$ be the closure of a neighborhood of $x$. No matter how large $i$ is taken, $O\left(x_{i}\right)$ intersects $X-N$ since $f$ is unstable. As in the proof of (2.1), let $m_{i}$ be the smallest integer in absolute value for which

$$
f^{m_{i}}\left(x_{i}\right) \notin N \text {. }
$$

Either an infinite number of the $m_{i}$ 's are positive or an infinite number are negative, or both. Assume that an infinite number are positive. If this is not the case, the proof is similar. We can select a sequence of these integers that go to infinity with their subscripts and such that the points $f^{m_{i}}\left(x_{i}\right)$ converge to a point $y \in X-N$. The negative semi-orbit of $y$ is asymptotic to $x$ by arguments similar to those used in the proof of (2.1).

(2.4) THEorem. If $x \in X$ is periodic under $f$, then there exists a point of $X$ whose orbit is asymptotic to $O(x)$ in at least one sense.

Proof. If $x$ is of period $m, x$ is a fixed point under $f^{m}(X)$. By (2.2), $f^{m}(X)$ is unstable and by the previous lemma there is a point $y \in X$ such that $O(y)$ is asymptotic (with respect to $f^{m}(X)$ ) to $x$ in at least one sense. Assume $O(y)$ is asymptotic to $x$ under $f^{m}(X)$ in the positive sense (the other possibility can be similarly treated). Then for $\epsilon>0$ given, there exists an integer $K_{0}$ such that $i>K_{0}$ implies

$$
d\left(f^{i m}(y), f^{i m}(x)\right)<\epsilon .
$$

Moreover, for a given integer $j$ there exists an integer $K_{j}$ such that $i>K_{j}$ implies

$$
d\left(f^{i m+i}(y), f^{i m+i}(x)\right)<\epsilon .
$$

If $K=\max \left(K_{0}, K_{1}, \cdots, K_{m-1}\right)$, then for $i>K$,

$$
d\left(f^{i m+j}(y), f^{i m+j}(x)\right)<\epsilon, \quad j=0,1,2, \cdots, m-1 .
$$

Thus there exists an integer $N$ (namely $(K+1) m)$ such that $n>N$ implies

$$
d\left(f^{n}(y), f^{n}(x)\right)<\epsilon .
$$

This completes the proof of the theorem.

Let $X$ be a metric space and let $f(X)=X$ be a homeomorphism. The point $x \in X$ will be called an unstable point under $f$ provided there exists a number $\delta(x)>0$ such that if $y$ is any point of $X$ distinct from $x$, then for some integer $n(y)$,

$$
d\left(f^{n}(x), f^{n}(y)\right)>\delta .
$$


It is easily seen that if $x$ is unstable under $f$, then each point of $O(x)$ is unstable under $f$ (for the same $\delta$ ). The following statements follow from the proofs of (2.2), (2.3), and (2.4).

(i) If $X$ is compact and metric and $x \in X$ is an unstable point under the homeomorphism $f(X)=X$, then for any integer $m \neq 0, x$ is unstable under $f^{m}(X)=X$.

(ii) If $x$ is a limit point of the compact metric space $X$ and is unstable and periodic under the homeomorphism $f(X)=X$, then there is a point of $X$ whose orbit is asymptotic to $O(x)$ in at least one sense.

The homeomorphism of the unit interval onto itself defined by $x \rightarrow x^{3}$ is an example of a transformation under which some, but not all, points are unstable.

\section{Additional results and examples.}

(3.1) Theorem. Let $X$ be compact and metric and let $f(X)=X$ be an unstable homeomorphism. If $K$ is any positive integer, the points of $X$ of period $K$ are finite in number. Thus the periodic points of $X$ form a countable set.

Proof. If $X$ contains an infinity of fixed points, they have a limit point which is a fixed point. At this fixed point instability is violated. If $X$ contains an infinity of points of period $K$, under $f^{K}(X)=X$ there are an infinity of fixed points, contrary to the instability of $f^{K}$. Thus the points of period $K$ are finite and the theorem is proved.

A compact dense-in-itself space has the potency of the continuum $[5$, p. 68]. Thus, by (3.1), there is no pointwise periodic unstable homeomorphism on such a space.

A transformation $\phi(M)=M$ is said to be regular on $M$, a metric space, if given a real number $\epsilon>0$, there exists a real number $\delta>0$ such that if $x$ and $y$ are points of $X$ for which $d(x, y)<\delta$, then $d\left(\phi^{n}(x), \phi^{n}(y)\right)<\epsilon$ for all integers $n$ (that is, $\phi$ has equi-continuous powers). A point $x$ of a metric space $X$ is said to be almost periodic under the homeomorphism $\phi(X)=X$ if given any real number $\epsilon>0$, there exists a relatively dense (successive differences bounded) sequence of integers $\left\{n_{i}\right\}$ unbounded above and below such that $d\left(\phi^{n_{i}}(x), x\right)<\epsilon$ for all integers $i$. The transformation $\phi$ is said to be almost periodic if, for each $\epsilon>0$, every point of $X$ is almost periodic under $\phi$ for the same sequence $\left\{n_{i}\right\}$. Clearly, instability is a strong contradiction of regularity on a space that is dense-in-itself. It is known [2] that if $\phi$ is almost periodic on a compact metric space, then $\phi$ is regular. Thus an unstable transformation on a compact, metric space that is dense-in-itself cannot be almost periodic. However, in 
the examples cited below there is an unstable homeomorphism that is pointwise almost periodic on such a space.

Examples of orbits asymptotic under unstable homeomorphisms can be found in the symbol spaces extensively investigated by Morse and Hedlund. ${ }^{3}$ If $\&$ is the space based on two symbols, then $\&$ is compact, metric, and dense-in-itself. Hedlund [3] has pointed out a pair of doubly asymptotic orbits in (E. Another example consists of the closure, $M$, of the orbit $T[4$, p. 832] due to Morse. This subset of $\&$ is a minimal set on which the transformation is pointwise almost periodic. In $M$ there is an orbit $T^{\prime}[4, \mathrm{p} .833]$ positively asymptotic to $T$, an orbit $S$ negatively asymptotic to $T$, and still a fourth orbit negatively asymptotic to $T^{\prime}$ and positively asymptotic to $S$. Other examples can be constructed in $\mathbb{E}$.

\section{REFERENCES}

1. M. Garcia and G. A. Hedlund, The structure of minimal sets, Bull. Amer. Math. Soc. vol. 54 (1948) pp. 954-964.

2. W. H. Gottschalk, Almost periodicity, equi-continuity and total boundedness, Bull. Amer. Math. Soc. vol. 52 (1946) pp. 633-636.

3. G. A. Hedlund, Sturmian minimal sets, Amer. J. Math. vol. 66 (1944) pp. 605620.

4. M. Morse and G. A. Hedlund, Symbolic dynamics, Amer. J. Math. vol. 60 (1938) pp. 815-866.

5. W. Sierpinski, Introduction to general topology, Toronto, 1934.

UNIVERSITY OF MISSOURI

3 and the unstable homeomorphism of $\mathfrak{E}$ onto itself are defined, for example, in $[1],[3]$, and [4]. 